\title{
La référence démonstrative dans les corpus écrits : théorisation et analyse de données empiriques
}

\author{
Vanderbauwhede, Gudrun \\ Katholieke Universiteit Leuven (Université de Louvain), Belgique \\ gudrun.vanderbauwhede@arts.kuleuven.be
}

\section{Introduction}

Le domaine de la référence démonstrative fait partie des thèmes de recherche qui ont fait durant des décennies et qui font toujours l'objet de nombreuses études dans plusieurs disciplines. Ainsi, pour ne citer que quelques axes de recherche et quelques traditions, souvent à situer à des niveaux très divers, la référence démonstrative a été décrite au sein de la référence définie et par rapport à la référence indéfinie selon des approches plutôt formelles ${ }^{1}$, sémantiques ${ }^{2}$, discursives ${ }^{3}$ et fonctionnalistes ${ }^{4}$. L'opposition entre les systèmes du déterminant démonstratif et de l'article défini a fait l'objet d'études tantôt synchroniques, souvent à dominante sémantique ${ }^{5}$ ou cognitive ${ }^{6}$, tantôt diachroniques ${ }^{7}$. Nous disposons également de nombreuses études synthétiques, parfois typologiques, des emplois référentiels ${ }^{8}$ et (inter)subjectifs ${ }^{9}$ du syntagme nominal démonstratif (SNdém) et de nombreuses études analytiques de certains emplois spécifiques, parfois insolites, du SNdém ${ }^{10}$. Enfin, le domaine du démonstratif a plus récemment été analysé sous un angle contrastif ${ }^{11}$, parfois à visée pédagogique ${ }^{12}$.

Le présent travail, basé sur l'analyse de données authentiques issues du Dutch Parallel Corpus (Paulssen et al. 2006) et du Corpus de Namur (Paulussen 1999), montrera, d'une part, que plusieurs clarifications terminologiques, conceptuelles et descriptives concernant les emplois référentiels du SNdém semblent s’imposer malgré l'état de la question impressionnant, ce qui peut aboutir à un nouvel aperçu synthétique de ces emplois (Section 2), et, d'autre part, qu'une analyse contrastive du déterminant démonstratif en français et en néerlandais contemporains, basée sur ce nouvel aperçu, permet de trouver plusieurs indices pour renforcer la piste diachronique de la désémantisation du SNdém français (e.a. Harris 1977, Jonasson 1998, De Mulder Carlier 2006) (Section 3).

\section{Réflexions théoriques autour des emplois référentiels du SNdém}

\subsection{Quatre regroupements existants}

Les études de Himmelmann (1996), de Diessel (1999), de Levinson (2004) et de Cornish (2010) font le point sur plusieurs classifications des emplois référentiels du SNdém. Ces auteurs distinguent en gros quatre emplois référentiels majeurs du SNdém. Ainsi, le SNdém sert à localiser le référent dans la situation de communication (emploi situationnel) (1) ; le SNdém renvoie de façon coréférentielle au co-texte linguistique (emploi anaphorique) (2); le SNdém renvoie de façon non coréférentielle à des entités 
linguistiques de nature diverse ou à des phénomènes abstraits dans le co-texte linguistique antérieur (propositions ou événements) (deixis discursive) (3) ; et enfin, l'identification du référent visé par le SNdém mémoriel s’appuie sur des connaissances partagées (emploi mémoriel) (4).

(1) Quelle heure est-il ? Non, ne me le dis pas, j'ai toujours su l'heure même en dormant; il doit être trois heures et quelques minutes. Les anges ont dû déjà faire la moitié du travail. Ils vont toujours par deux. Cela surtout pour le transport de l'âme. En fait l'un se pose sur l'épaule droite, l'autre sur la gauche et dans un même élan, un mouvement lent et gracieux, ils emportent l'âme vers le ciel. Mais cette nuit, ils nettoient. (CdN - Textes fictionnels)

(2) Le temps opératoire est plus court avec le TOT. Cette dernière technique a aussi l'avantage de pouvoir être réalisée chez des femmes qui ont déjà eu des interventions chirurgicales dans le petit bassin et qui présentent d'importantes adhérences.

(DPC - Textes informatifs)

(3) Dis-lui un mot pour moi, mon petit vieux, me lança Alan après que je l'eus quitté. Dis-lui que dans tous mes rêves libidineux je lui assigne un rôle d'esclave sexuelle. Pourrait-elle faire quelque chose à ce propos? (CdN - Textes fictionnels)

(4) Tous se trouvaient ce soir-là dans cet état d'extrême fatigue qui confine à l'ivresse, unis dans la satisfaction du travail accompli, de la récolte rentrée, baignés par ce crépuscule de fin juillet qui ne se décidait pas à faire place à la nuit, comme [...].

(CdN - Textes fictionnels)

Le schéma de Himmelmann (1996) résume les quatre emplois référentiels majeurs du SNdém dans une autre figure asymétrique. Selon Himmelmann (1996), les SNdém peuvent établir un référent dans l'univers du discours pour la première fois (introducing : emploi situationnel, deixis discursive) ou sélectionner un référent parmi d'autres référents déjà établis (singling out : emploi anaphorique, emploi mémoriel). Himmelmann (1996) insiste également sur les rapprochements possibles entre les différents types d'emplois, bien qu'il ne les traite pas tous dans son analyse. Le SNdém peut, par exemple, donner une lecture mémorielle à un référent plus ou moins établi dans le co-texte ou le discours linguistique antérieur (rapprochement de l'emploi mémoriel et de l'emploi anaphorique). Le SNdém peut également renvoyer à des éléments dans le contexte situationnel dont il a déjà été question dans le discours linguistique antérieur (rapprochement de l'emploi situationnel et de l'emploi anaphorique). 


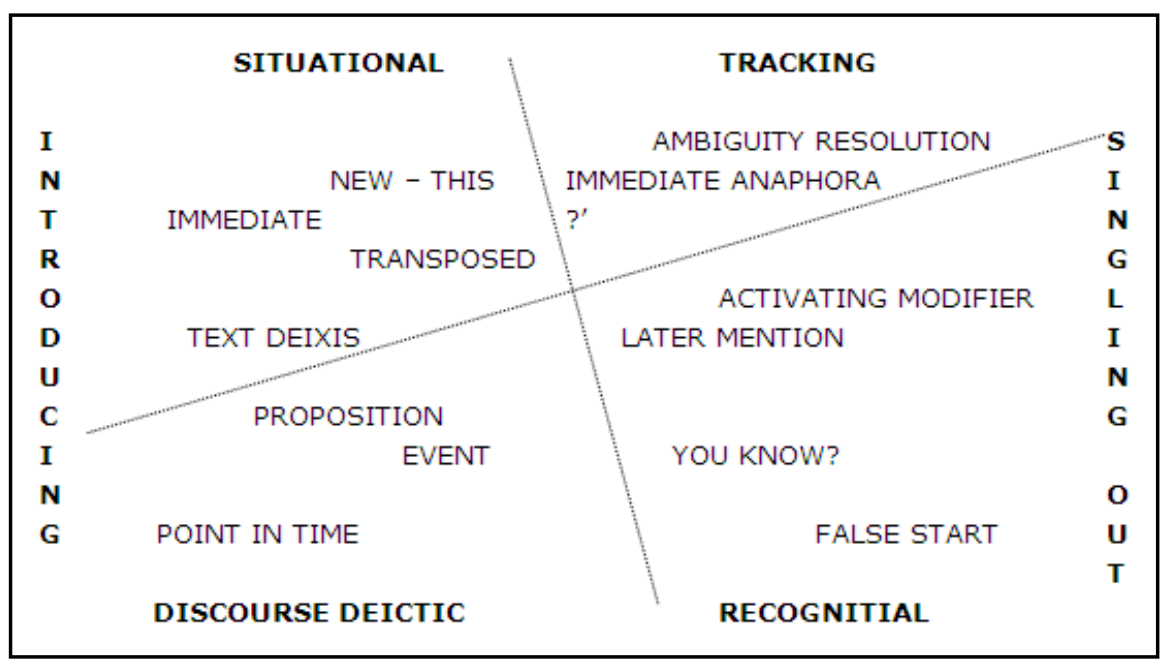

Figure 1 : Les emplois référentiels majeurs du SNdém selon Himmelmann (1996 : 241)

Diessel (1999), pour sa part, fait la distinction entre les emplois exophoriques et les emplois endophoriques, suite à Halliday - Hasan (1976). Par exophorique, il entend l'emploi situationnel du démonstratif; par endophorique, il entend tous les autres emplois du démonstratif, à savoir l'emploi anaphorique, la deixis discursive et l'emploi mémoriel.

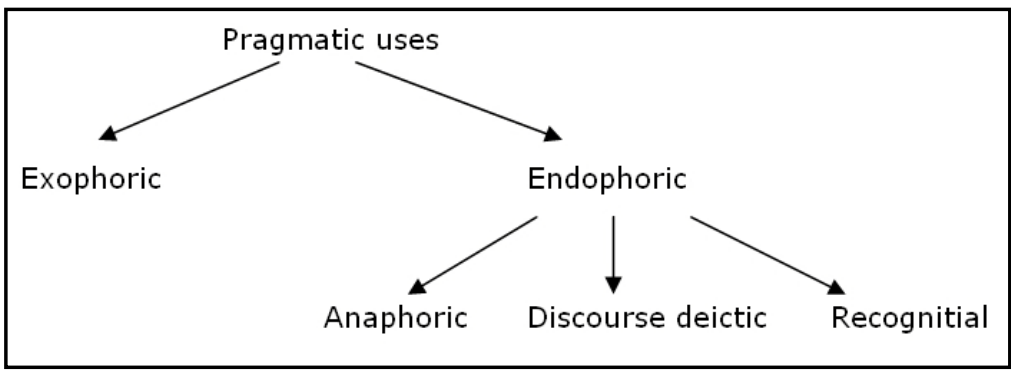

Figure 2 : Regroupement des emplois référentiels majeurs du SNdém selon Diessel (1999 : 6)

Alors que Diessel (1999) propose un regroupement asymétrique, Levinson (2004) inclut tant l'emploi situationnel que la deixis discursive dans les emplois déictiques et regroupe l'emploi anaphorique et l'emploi mémoriel dans les emplois non-déictiques. Levinson réserve également une place à l'emploi (inter)subjectif du démonstratif (empathetic use). 


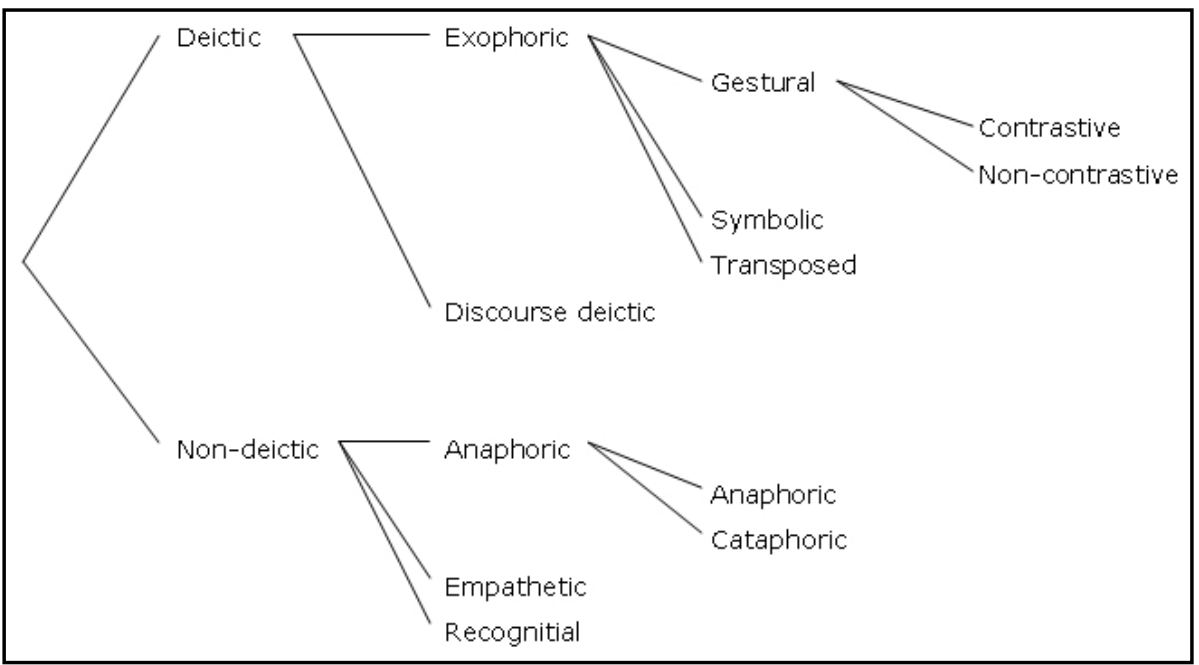

Figure 3 : Regroupement des emplois référentiels du SNdém selon Levinson (2004 : 108)

Cornish (1999, 2001, 2007, 2008, 2010), enfin, propose le terme d'anadeixis (Ehlich 1982) pour indiquer le type de référence indexicale qui combine des procédés anaphoriques et situationnels à divers degrés. Ainsi, la deixis discursive et l'emploi mémoriel contiennent à la fois des procédés textuels et des procédés situationnels.

\begin{abstract}
The indexical expressions which realise anadeixis are anaphoric in that their referent is already (potentially) present in the discourse representation assumed by the speaker to be shared by speaker and addressee at the point of occurrence, and is retrieved via this reference. It is deictic in that the speaker is having recourse to the utterance context to (re)direct the addressee's attention focus to a referent which, although potentially available within the discourse context at the time of utterance, is not the one to which subsequent reference would be expected to be made at that point. It is not canonically deictic, in that there is no totally new referent being introduced into the discourse thereby, and not all the utterance-level parameters are being altered via this reference. (Cornish 2010)
\end{abstract}

Selon l'échelle de Cornish, la deixis discursive et l'emploi mémoriel se situent, par conséquent, entre l'emploi situationnel ou déictique et l'emploi anaphorique.

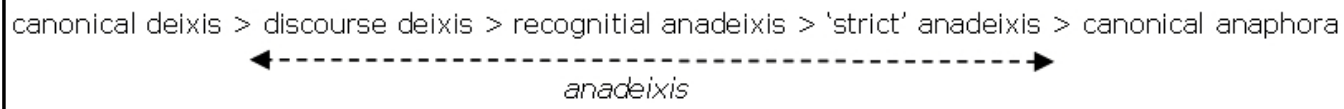

Figure 4 : Échelle de procédures référentielles indexicales (Cornish 2010)

Les mérites de ces regroupements sont multiples. Si l'aperçu typologique de Diessel (1999) est encore relativement simple et fait seulement état des quatre emplois référentiels majeurs du SNdém, le schéma de Levinson (2004) est déjà plus détaillé et inclut l'opposition de base entre les emplois qui renvoient au contexte situationnel et ceux qui ne renvoient pas au contexte situationnel. Par ailleurs, le mérite le plus important des regroupements de Himmelmann (1996) et de Cornish (2010) consiste sans aucun doute dans le rapprochement des différents emplois référentiels du SNdém. Ainsi, tant Himmelmann (1996) que Cornish (2010) insistent sur le fait qu'il existe plusieurs zones de transition importantes entre les quatre emplois référentiels majeurs du SNdém et notamment entre l'opposition renvoi au contexte situationnel - renvoi au co-texte linguistique. 


\subsection{Proposition d'un nouvel aperçu synthétique}

Cependant, nous constatons que la définition et la délimitation de l'emploi référentiel du SNdém et des quatre sous-domaines d'emplois sont problématiques sur certains points et qu'il se pose notamment des questions en matière de délimitation des classes d'emplois pour ce qui est de la deixis discursive et de l'emploi mémoriel du SNdém. De plus, l'articulation des différents emplois référentiels mérite réflexion, notamment à la lumière de la double conception de la définitude, pragmatique et sémantique, proposée dans la littérature. Selon Löbner (1985), l'on parle de définitude pragmatique quand le SN introduit par l'article défini ou par le déterminant démonstratif dépend du contexte, tandis que la définitude sémantique se caractérise par le fait que le référent du SN n’est pas directement accessible pour l'interlocuteur, étant donné qu'il n'est pas donné dans le contexte d'énonciation. Contrairement à la définitude pragmatique, qui implique que l'interlocuteur peut repérer le référent en se fondant sur le contexte, la définitude sémantique s'applique aux cas où l’identification du référent n'est pas ajustée dans la parole ou dans le discours, comme c'est le cas, par exemple, des emplois génériques purs.

Pragmatically definite NPs are essentially dependent on special situations and contexts for the nonambiguity of a referent. (Löbner 1985: 298)

Semantic definites : the referent of the definite is established independently of the immediate situation or context of utterance. [...] Semantic definites refer unambiguously due to general constraints. (Löbner 1985: 298-299)

Notre aperçu synthétique des emplois référentiels du SNdém (Figure 5) tente de proposer une solution aux différents problèmes de définition et de délimitation.

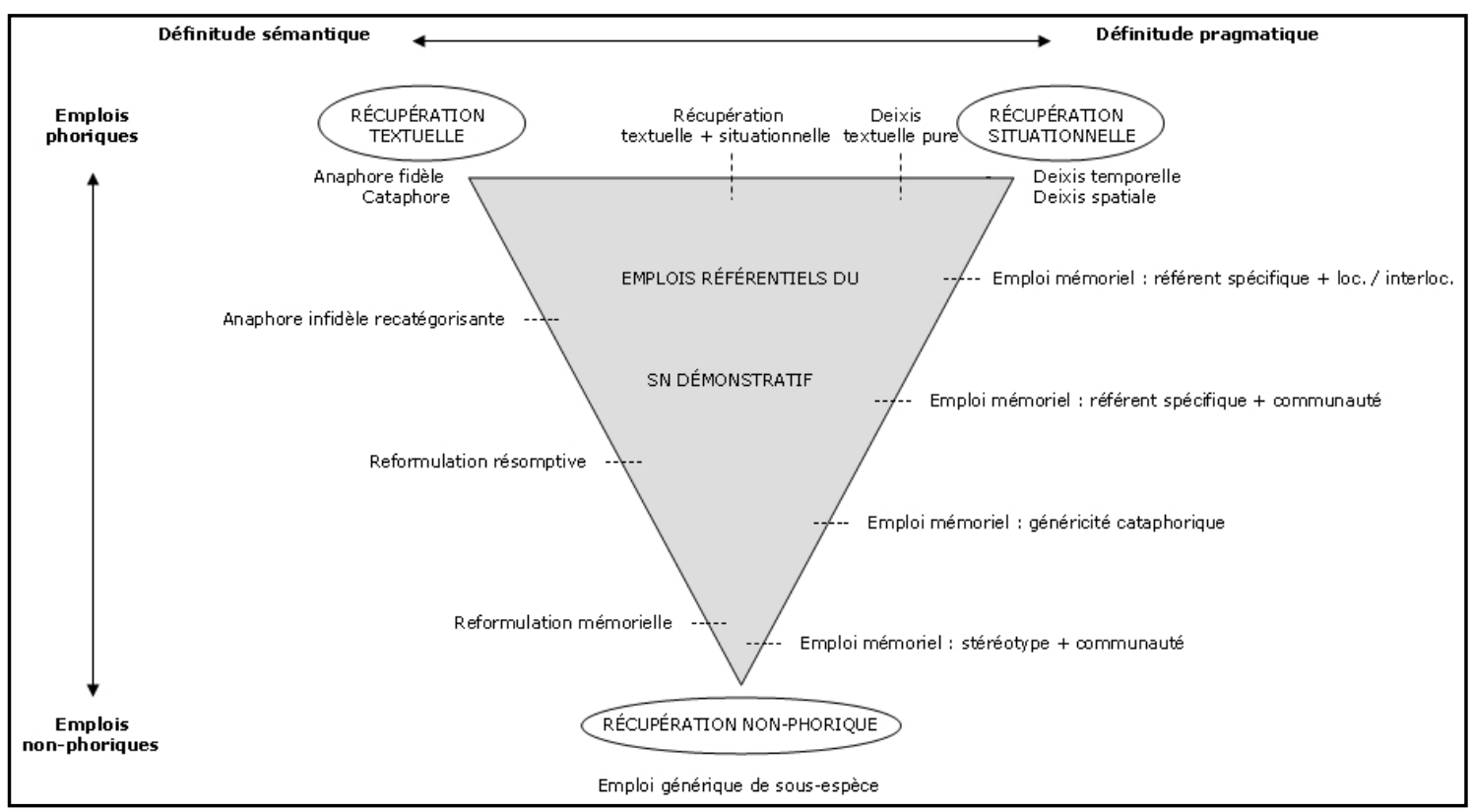

Figure 5 : Aperçu synthétique des emplois référentiels du SNdém

En premier lieu, nous estimons avec Breban (2010) et Davis - Potts (2009) que les emplois (inter)subjectifs du SNdém (cf. deixis émotive, deixis emphatique, démonstratif 
affectif) doivent être considérés comme des emplois qui se greffent sur les emplois référentiels de base du SNdém, contrairement à ce que suggère l'aperçu synthétique de Levinson (2004). Dans l'exemple suivant, par exemple, différentes stratégies romanesques (discours direct, présent historique) semblent créer les conditions propices à l'apparition de l'emploi déictique émotif du SNdém. Nous estimons pourtant que les SNdém ne réfèrent pas complètement in absentia (i.e. pas de renvoi situationnel ou textuel), mais qu'il y a un lien anaphorique assez clair entre fumée et brûler, d'une part, et odeur et eau-de-vie, d'autre part, à côté d'un léger effet de sens mémoriel (l'événement est vécu par l'auteur, sans qu’il soit nécessairement partagé par le lecteur).

(5) Nous allons le soir au café ; on est trois ou quatre anciens camarades; on joue sa demi-tasse, son petit verre et l'on fait brûler son eau-de-vie! Cette fumée, cette odeur d'alcool, le bruit des billes, le saut des bouchons, les gros rires, tout cela double mes sens et il me semble qu'il m'est poussé des moustaches et que je soulèverais le billard!

(Apothéloz 1995 : 35)

Cet exemple montre qu'il est pratiquement impossible d'exclure complètement l'emploi référentiel du SNdém de sa valeur (inter)subjective. En plus, du point de vue diachronique, l'analyse de Breban (2010) confirme que les emplois (inter)subjectifs du SNdém sont ultérieurs aux emplois référentiels en anglais. Du point de vue typologique, les données de Davis - Potts (2009) confirment que les emplois (inter)subjectifs du SNdém sont récurrents dans diverses langues, qu'ils ne relèvent pas d'une ambiguïté d'emplois, mais qu'ils doivent être considérés comme une extension naturelle des emplois référentiels. Voilà pourquoi il nous semble préférable de ne pas traiter au même niveau la valeur référentielle du SNdém et sa valeur (inter)subjective.

En deuxième lieu, nous constatons qu'un autre problème de définition et de délimitation a trait à la catégorie de la deixis discursive. En effet, le contenu de la classe de la deixis discursive et des emplois qui en ont été rapprochés dans la littérature (e.a. Lyons 1977, Levinson 1983, Himelmann 1996, Fillmore 1997, Cornish 1999, Diessel 1999) a un caractère opaque et hétérogène qui prête à confusion et est éparpillé sur l'ensemble de notre aperçu synthétique. Ainsi, nous faisons la distinction entre la deixis textuelle (pure), évoquant divers effets situationnels (6), métadiscursifs (7) et métalinguistiques (8), la reformulation résomptive qui se trouve sur la frontière de la récupération textuelle et la récupération non-phorique (9) et un emploi se situant sur la frontière de la récupération textuelle et situationnelle (i.e. procédés textuels et situationnels mixtes) (10).

(6) Avant propos. Ce livre se veut bien plus que la biographie d'une des grandes figures emblématiques de la politique libérale belge et européenne de ces cinquante dernières années. (DPC - Textes non-fictionnels)

(7) 'Cette' : ce mot est un déterminant démonstratif. (exemple forgé)

(8) Écoute cette histoire : j'ai vu un monsieur à Paris, et ce monsieur [...]. (exemple forgé)

(9) Monsieur le Président, si nous avons bien compris, la Commission fera une déclaration sur la Bosnie mercredi, mais sans possibilité pour le Parlement de déposer de résolutions et sans temps de parole supplémentaire par rapport à ce qui est prévu. Certes, nous comprenons 
l'embarras qui est le vôtre, Monsieur le Président au regard du risque de voir se répéter le triste spectacle qu'a donné notre Parlement à ce sujet lors de la session d'avril. (CdN - Débats écrits)

(10) Regardez à présent ce chapelet ... du corail, de l'ambre, de l'argent ... Il a dû appartenir à un imam. Peut-être que la femme le portait comme collier ... Des pièces de monnaie ... un rial troué ... un centime ... un franc marocain ... Des billets de banque qui n'ont plus de valeur ... Un dentier ... Une brosse ... Un bol en porcelaine ... Un album de cartes postales ... J'arrête de sortir ces objets ...

(CdN - Textes fictionnels)

En troisième lieu, notre aperçu systématique montre que la plus grande partie des emplois mémoriels du SNdém sont à situer sur le côté qui va de la récupération situationnelle à la récupération non-phorique. D’une part, notre typologie des emplois mémoriels du SNdém met l'accent sur la distinction entre les connaissances partagées spécifiques et personnelles et celles qui portent sur la mémoire collective. D’autre part, nous prêtons également attention à la distinction entre un référent stéréotypique (11), un référent basé sur une sous-classe générique (12) et un référent spécifique (13), en mettant à profit certaines propositions de Kleiber (2005, 2008). Alors que les emplois mémoriels présentés dans les exemples (11) à (13) se situent sur le côté droit du triangle, la reformulation mémorielle (14), incluant également des procédés anaphoriques textuels, se situe sur le côté gauche du triangle.

(11) «Je me pose constamment la question : est-ce qu'on retrouve le caractère volontaire, fiable, unique et chaleureux dans ce slogan, cette photo, cette campagne? ", explique Peggy De Coeyer.

(DPC - Textes journalistiques)

(12) Il fait partie de ces hommes qui, par tradition familiale plus que par mode, a pris la direction du Droit.

(DPC - Textes non-fictionnels)

(13) Vous vous souvenez de ces images qui ont fait le tour du monde, où l'on vous voit en train de vous doper?

(DPC - Textes journalistiques)

(14) J'ai connu 'la lucide férocité des meilleurs jours', ces jours où tout semble paisible. (CdN - Textes fictionnels)

En dernier lieu, nous avons essayé d'intégrer maximalement la dichotomie de la définitude pragmatique et de la définitude sémantique en relation avec les emplois phoriques et non-phoriques du SNdém dans notre aperçu systématique. Tout d'abord, la Figure 5 montre que l'emploi textuel du SNdém, avec l'anaphore fidèle comme emploi textuel prototypique, relève de la définitude sémantique, contrairement à ce que suggère le classement de Löbner (1985). Ensuite, nous constatons que certains emplois, tels que l'emploi mémoriel, l'emploi générique de sous-espèce et l'emploi incluant des procédés référentiels textuels et situationnels du SNdém se trouvent dans une zone intermédiaire entre la définitude pragmatique et sémantique. La définitude pragmatique n’exclut donc pas nécessairement la définitude sémantique et vice versa. Enfin, l'aperçu montre que seul l'emploi situationnel du SNdém, renvoyant au contexte d'énonciation, relève de la définitude pragmatique. Cela dit, il convient d'affirmer que la sémantique en termes de sens des mots, des phrases, etc. intervient évidemment toujours, même au niveau des emplois pragmatiquement définis les plus forts du SNdém, et que les emplois 
sémantiquement définis du SNdém présentent eux aussi leurs propres stratégies ou exploitations discursives.

\title{
3 La désémantisation du SNdém français : de nouveaux indices à partir d'une étude contrastive français - néerlandais
}

L’intégration des différents emplois attestés dans la littérature et la clarification de certains problèmes terminologiques n'était pas la seule motivation de cette étude pour créer un nouvel aperçu synthétique et unifié des emplois référentiels du SNdém. Il devait ensuite directement servir de point de départ à l'étude contrastive basée sur corpus des emplois référentiels du SNdém en français contemporain et en néerlandais contemporain dans le cadre d'une analyse plus approfondie de l'hypothèse diachronique de la désémantisation du SNdém.

\subsection{L'hypothèse de la désémantisation du SNdém français}

Plusieurs auteurs (e.a. Harris 1977, Greenberg 1978, De Mulder - Carlier 2006) se sont intéressés à l'existence d'un cycle qui mène du démonstratif à l'article défini. Greenberg (1978) propose le cycle menant du démonstratif à l'article défini et au marqueur nominal suivant.

\begin{tabular}{|cccc|}
\hline Stage 0 & Stage 1 & Stage 2 & Stage 3 \\
Demonstrative & $>$ & Specific article & $>$ \\
Definite article & Noun marker
\end{tabular}

Figure 6 : Le cycle de l'évolution du démonstratif de Greenberg (Greenberg 1978)

Du stade 0 au stade 1, l’on passe du démonstratif à l'article défini générique (Greenberg 1978 : 61-62). Ensuite, du stade 1 au stade 2, l'on passe de l'article défini à l'article spécifique ou non-générique. L'article du stade 2 montre les premiers emplois de l'article indéfini au singulier ${ }^{13}$. Enfin, du stade 2 au stade 3, l'on passe de l'article spécifique ou non-générique au marqueur nominal. L’article du stade 3 est un marqueur nominal auquel sont associées les catégories morphologiques du genre et du nombre.

De Mulder - Carlier (2006) appliquent le cycle proposé par Greenberg (1978) à l'évolution du démonstratif du latin au français et à la réorganisation du système du démonstratif en français.

\begin{abstract}
À partir du démonstratif de l'éloignement ille du latin naît en ancien français l'article défini li. Parallèlement, à partir des formes renforcées du démonstratif ecce ille et ecce iste du latin tardif se développent en ancien français les formes cil et cist, prenant la relève pour assumer la fonction démonstrative. Leur affaiblissement conduit à l'apparition de la forme atone ce. Cet affaiblissement est à nouveau corrélé au développement des formes renforcées ce $\mathrm{N}$-ci et ce $\mathrm{N}$-là. (De Mulder - Carlier 2006 : 96)
\end{abstract}

La question qui se pose est celle de savoir à quel stade du cycle se trouve le déterminant démonstratif du français moderne et ce qu'il en est de l'article défini. Selon Harris (1977), l'article défini en français moderne a atteint le dernier stade du cycle de Greenberg (1978), celui de marqueur nominal, et le déterminant démonstratif simple a atteint le stade initial de l'article défini, ne distinguant plus l'opposition proximité - 
distance. Seuls les déterminants démonstratifs composés sont encore de véritables démonstratifs.

\begin{tabular}{|c|c|c|c|}
\cline { 2 - 4 } \multicolumn{1}{c|}{} & $\begin{array}{c}\text { Prefix markers of } \\
\text { number and gender }\end{array}$ & Articles & De monstratives \\
\hline Classical Latin & - & - & hic ; iste; ille \\
\hline Vulgar Latin & - & ille & ecce iste; ecce ille \\
\hline Old French & - & cest ; cel \\
\hline Modern French & ie & ce & ce ... ci; ce ... is \\
\hline
\end{tabular}

Figure 7: L'évolution de l'article défini et du démonstratif en français selon Harris (Harris 1977 : 256)

L'analyse de De Mulder - Carlier (2006), par contre, permet de conclure que le déterminant démonstratif $c e$ s'est effectivement engagé dans un processus qui conduit vers l'article défini, mais que « les chances d'accéder au statut d'article défini sont plus réduites pour le démonstratif français ce qu'elles n'étaient pour le démonstratif latin ille ", étant donné que le déterminant démonstratif en français moderne est toujours ancré dans son contexte d'emploi (situationnel ou textuel) (De Mulder - Carlier 2006 : 110-111). L'étude de De Mulder - Carlier (2006), enfin, permet de conclure que même l'article défini le est "loin d'être un simple marqueur du statut nominal et des catégories morphologiques du genre et du nombre qui y sont associées » (De Mulder Carlier 2006 : 111), ce qui va à l'encontre de l'étude de Harris (1977).

Dans ce qui suit, nous étudions plus en détail le rapprochement du déterminant démonstratif $c e$ et de l'article défini le en français contemporain. Pour ce faire, nous comparons le fonctionnement formel et sémantique des emplois référentiels du SNdém en français et en néerlandais contemporains, afin de montrer que le déterminant démonstratif français est instructionnellement plus faible que le déterminant démonstratif néerlandais. Notre étude contrastive basée sur des données synchroniques essaie ainsi de fournir des indices supplémentaires pour l'hypothèse diachronique de la désémantisation du SNdém français.

\subsection{Résultats quantitatifs}

L'étude du déterminant démonstratif dans deux corpus comparables du français et du néerlandais, basés sur le Dutch Parallel Corpus et sur le Corpus de Namur, nous permet tout d'abord de localiser les cas de divergence au niveau de leur emploi entre les deux langues et d'en mesurer l'importance quantitative. 


\begin{tabular}{|c|c|c|c|c|}
\hline \multirow[t]{2}{*}{ Emploi référentiel du SNdém } & \multicolumn{2}{|c|}{ SNdém FR } & \multicolumn{2}{|c|}{ SNdém NL } \\
\hline & n & $\%$ & n & $\%$ \\
\hline Récupération textuelle & 671 & 68.61 & 549 & 72.33 \\
\hline Anaphore fidèle & 170 & 17.38 & 196 & 25.82 \\
\hline Sans changement de nombre & 57 & 5.83 & 89 & 11.73 \\
\hline Avec passage au pluriel & 3 & 0.31 & 4 & 0.53 \\
\hline Avec passage au singulier & 5 & 0.51 & 2 & 0.26 \\
\hline Avec retranchement d'informations & 88 & 9.00 & 87 & 11.46 \\
\hline Avec ajout d'informations & 17 & 1.74 & 14 & 1.85 \\
\hline Cataphore & 9 & 0.92 & 2 & 0.26 \\
\hline Anaphore infidèle recatégorisante & 239 & 24.44 & 141 & 18.58 \\
\hline Synonyme & 86 & 8.79 & 63 & 8.30 \\
\hline Hyponyme & 0 & 0 & 0 & 0 \\
\hline Hyperonyme & 64 & 6.54 & 44 & 5.80 \\
\hline Nom de qualité & 17 & 1.74 & 4 & 0.53 \\
\hline Ajout d'informations & 72 & 7.36 & 30 & 3.95 \\
\hline Reformulation résomptive & 253 & 25.87 & 210 & 27.67 \\
\hline Emploi générique de sous-espèce & $\mathbf{0}$ & $\mathbf{0}$ & $\mathbf{0}$ & $\mathbf{0}$ \\
\hline Récupération mémorielle & 115 & 11.76 & 42 & 5.53 \\
\hline Reformulation mémorielle & 34 & 3.48 & 5 & 0.66 \\
\hline Emploi mémoriel & 81 & 8.28 & 37 & 4.87 \\
\hline Stéréotype + communauté & 13 & 1.33 & 6 & 0.79 \\
\hline Généricité cataphorique & 5 & 0.51 & 11 & 1.45 \\
\hline Référent spécifique + communauté & 2 & 0.20 & 0 & 0 \\
\hline Référent spécifique + loc. / interloc. & 61 & 6.24 & 20 & 2.64 \\
\hline Récupération situationnelle & 111 & 11.35 & 106 & 13.97 \\
\hline Deixis temporelle & 65 & 6.65 & 51 & 6.72 \\
\hline Deixis spatiale & 46 & 4.40 & 55 & 7.25 \\
\hline Deixis textuelle pure & 16 & 1.64 & 29 & 3.82 \\
\hline Récupération textuelle + situationnelle & 54 & 5.52 & 31 & 4.08 \\
\hline Superposition d'emplois & 11 & 1.12 & 2 & 0.26 \\
\hline TOTAL & 978 & 100 & 759 & 100 \\
\hline
\end{tabular}

Tableau 1 : La répartition des emplois référentiels des SNdém dans les deux corpus monolingues français et néerlandais

Ainsi, dans le Tableau 1, nous constatons des divergences importantes au niveau des SNdém anaphoriques : les SNdém anaphoriques fidèles représentent 17,38\% en français et 25,82\% en néerlandais, alors que les SNdém anaphoriques infidèles, pour leur part, représentent $24,44 \%$ des occurrences en français et $18,58 \%$ en néerlandais. De plus, nous relevons des différences assez importantes pour ce qui est des SNdém situationnels (11,35\% en français et $13,97 \%$ en néerlandais) et des SNdém déictiques textuels purs (1,64\% en français et 3,82\% en néerlandais). Cependant, la divergence quantitative la plus importante se situe au niveau des SNdém mémoriels avec 11,76\% de SNdém mémoriels français et seulement 5,53\% de SNdém mémoriels néerlandais. Notre aperçu synthétique des emplois référentiels du SNdém montre donc, grâce à l'intégration de la dimension définitude sémantique - pragmatique et de l'opposition emplois phoriques non-phoriques, que les SNdém français sont plus fréquents que les SNdém néerlandais dans la partie inférieure de l'aperçu, partie qui se rapproche du pôle de la récupération non-phorique ou générique. 


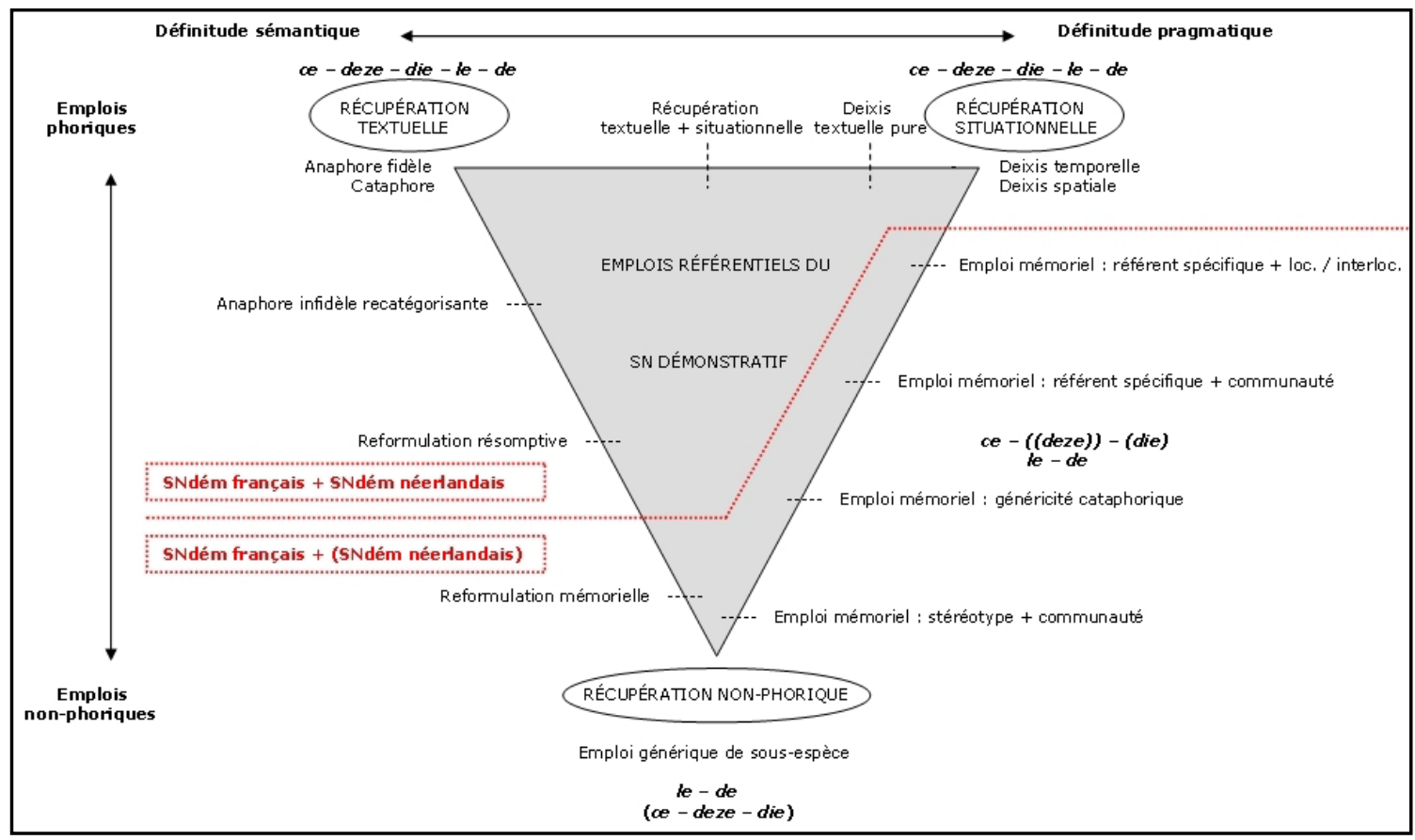

Figure 8 : La répartition globale des SNdém français et néerlandais dans l'aperçu synthétique

\subsection{Proposition d'hypothèses explicatives}

Nous proposons trois hypothèses explicatives majeures pour les différents cas de divergence relevés dans les corpus monolingues : des normes stylistiques différentes (3.3.1), des contraintes linguistiques plus générales (3.3.2) et un affaiblissement de la force instructionnelle du SNdém français (3.3.3). Nous verrons que la dernière hypothèse permet d'expliquer la plus grande partie des différences quantitatives.

\subsubsection{SNdém anaphoriques : différences stylistiques}

Tout d'abord, des différences stylistiques se trouvent à la base du constat selon lequel il y a moins de SNdém anaphoriques infidèles en néerlandais qu'en français dans notre corpus. En effet, nos résultats confirment le constat plus général fait dans d'autres études (p. ex. Skytte - Korzen 2000, Korzen - Lundquist (éds) 2003, Lundquist 2005) selon lequel l'emploi de l'anaphore infidèle serait plus répandu dans les langues romanes (p. ex. français, italien) que dans les langues germaniques (p. ex. néerlandais, danois). En d'autres mots, l'usage plus fréquent de l'anaphore infidèle en français s'explique en partie par le fait que le français varie davantage les renvois anaphoriques dans un texte, uniquement pour des soucis stylistiques. Cela semble être moins le cas en néerlandais. 


\subsubsection{SNdém anaphoriques et spatiaux : contraintes linguistiques plus générales}

Ensuite, nous constatons que certaines contraintes linguistiques ne sont pas imputables au démonstratif même, mais plutôt à des constructions plus englobantes dans lesquelles apparaît le démonstratif. Ces structures permettent également de mieux comprendre pourquoi nous avons trouvé moins de SNdém anaphoriques infidèles en néerlandais qu'en français. Ainsi, à la suite de Lundquist (2005), nous observons que l'emploi syntaxique de l'anaphore infidèle avec expansion lexicale diffère en français et en néerlandais. En effet, le $\mathrm{SN}$ français en position d'expression à gauche détachée est parfaitement transférable en anaphore infidèle avec expression à droite intégrée (15), contrairement au néerlandais (16). Parmi les autres SNdém anaphoriques infidèles sans expansion lexicale, qui sont également plus nombreux en français qu'en néerlandais, nous repérons deux structures ne contenant pas de déterminant démonstratif en néerlandais. Il s’agit, plus concrètement, du lexème spécialisé beide en néerlandais (both en anglais) qui se traduit souvent par ces deux en français (17) et de la tournure à un seul démonstratif en néerlandais dat is de Y die / waarin / waardoor ... qui peut se traduire par une structure clivée à deux démonstratifs en français, dont le premier est affaibli (c'est (X) ce Y que ...) (18).

(15) Premier renfort du club andalou, Aleksandr Kerzhatov a signé pour cinq ans et demi. Il aura pour nouvel équipier Aleksandr Kerzhatov. Ce premier renfort du club andalou a signé un contrat pour cinq ans et demi.

(16) *Eerste versterking van de Andalusische club, Aleksandr Kerzhatov tekende een contract voor vijfenhalf jaar.

Als nieuwe ploegmaat krijgt hij Aleksandr Kerzhatov. *Deze eerste versterking van de Andalusische club tekende een contract voor vijfenhalf jaar.

(17) Uit de verschillende presentaties blijkt dat beide chirurgische technieken als weinig invasief kunnen worden beschouwd.

Il ressort de ces différentes présentations que ces deux techniques chirurgicales peuvent être considérées comme peu invasives. (DPC - Textes informatifs)

(18) Het was niet verwonderlijk dat de eerste gedichten en verhalen die ik schreef Nederlandstalig waren. Dat was de taal waardoor ik in aanraking kwam met begrippen als stijl, analyse en plot.

Tout naturellement, les premiers poèmes et récits que j'ai écrits étaient en néerlandais. C'est par cette langue que j'ai découvert des notions telles que le style, l'analyse et l'intrigue d'un récit. (DPC - Textes non-fictionnels)

Pour ce qui est des divergences entre les SNdém spatiaux en français $(4,40 \%)$ et en néerlandais (7,25\%), telles que le Parlement vs. dit Parlement et la Commission vs. deze Commissie, nous avançons l'hypothèse selon laquelle les SN le Parlement et la Commission ont acquis le statut de noms propres avec article défini fixe en français, ce qui n'est pas encore le cas pour le néerlandais (19). Cette hypothèse porte sur le degré de figement de certaines structures et elle permet selon nous d'expliquer en partie le nombre plus élevé de SNdém spatiaux en néerlandais dans notre corpus.

(19) Mijnheer de Voorzitter, wij zullen in de maand juni weer over het probleem van BST, bovine somatotropine, spreken, aangezien dat eveneens in de discussie terug zal komen. Ik hoop dat wij 
dan meer geluk hebben dan de heer Vandemeulebroucke en dat wij dan dus antwoorden krijgen op vragen die al meer dan een jaar geleden door dit Parlement zijn gesteld.

Monsieur le Président, nous reparlerons du problème de la BST, somatotropine bovine, au mois de juin puisque cela reviendra également dans la discussion et j'espère à ce moment-là avoir plus de chance que M. Vandemeulebroucke et obtenir les réponses aux questions qui ont été posées par le Parlement voici plus d'un an maintenant.

(CdN - Débats écrits)

\subsubsection{SNdém anaphoriques, mémoriels, temporels et déictiques textuels purs : affaiblissement de la force instructionnelle du SNdém français}

Enfin, afin de pouvoir expliquer pourquoi nous avons trouvé tant de différences quantitatives dans nos corpus monolingues au niveau des emplois textuels, mémoriels, temporels et déictiques textuels purs et tant de SNdém français traduits par des SNdéf néerlandais dans le corpus parallèle, nous avançons l'hypothèse de l'affaiblissement de la force instructionnelle du SNdém en français par rapport à celle du SNdém en néerlandais.

Par force instructionnelle, nous entendons l'effort instructionnel véhiculé par le SNdém et le SNdéf afin de trouver le référent dans le co-texte antérieur et/ou dans le contexte situationnel ( $c f$. la deixis au sens large), de le désigner et de le mettre au premier plan (en focus). Nous empruntons l'idée de force instructionnelle à Kirsner $(1979,1993)$ qui propose une opposition de la haute deixis et de la basse deixis en termes d'instructions : la haute deixis donne une instruction plus forte pour trouver le référent dans le co- et/ou contexte ( " greater urging to the hearer to seek out and attend to the noun's referent, i.e. a more forceful instruction »), alors que la basse deixis donne une instruction plus faible pour trouver le référent dans le co- et/ou contexte (« lesser urging to the hearer to seek out and attend to the noun's referent, i.e. a less forceful instruction ») (Kirsner 1979 : 89). Ceci permet, par exemple, d'opposer la forme démonstrative proximale deze de la forme distale die en néerlandais.

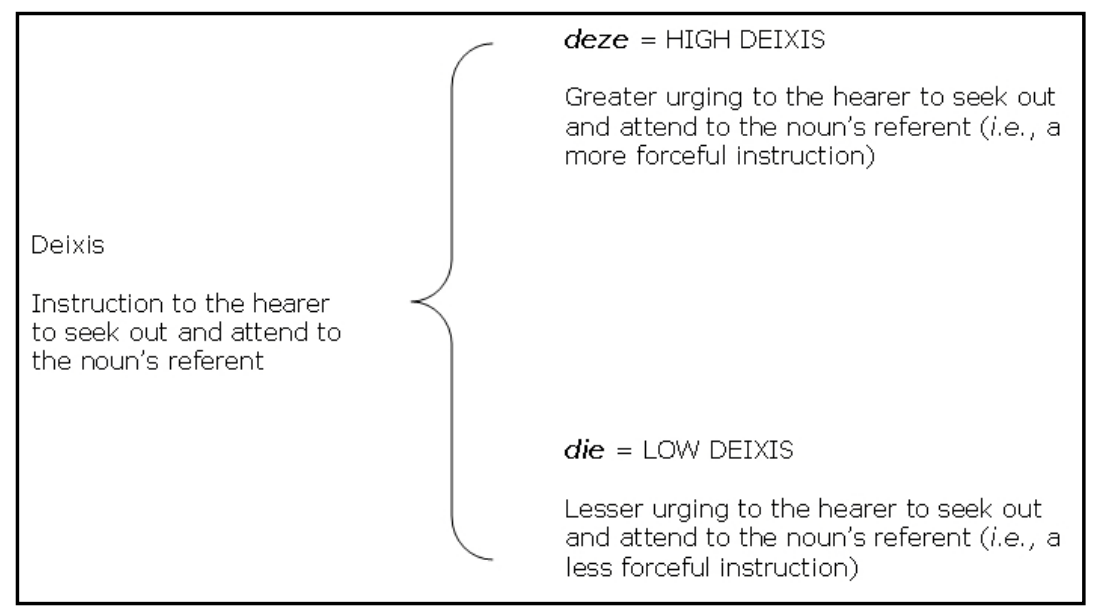

Figure 9 : L'opposition high deixis - low deixis (Kirsner 1979 : 89) 
Bien que Kirsner lie la force instructionnelle à nombre de paramètres permettant de distinguer la haute deixis de la basse deixis (p. ex. givenness, novelty in discourse, sharpness of referent, human NP, contrastive emphasis) (Tableau 2), nous prenons pour notre étude essentiellement en considération les paramètres de désignation dans le coet/ou contexte, de notification et de mise au premier plan pour décrire la force instructionnelle du SNdém et du SNdéf.

\begin{tabular}{|c|c|c|}
\hline Parameters & high deixis & low deixis \\
\hline note worthiness & more & less \\
\hline givenness & lesser & greater \\
\hline foregrounding & more & less \\
\hline distance to referent & greater, intersentential & lesser, intrasentential \\
\hline importance of referent & greater & lesser \\
\hline sharpness of referent & vivid & dull \\
\hline novelty in discourse & greater, new & lesser, old \\
\hline human NP & more & less \\
\hline contrastive emphasis & more & less \\
\hline text genre & government texts & novels \\
\hline spatiotemporal location & near & unmarked \\
\hline
\end{tabular}

Tableau 2 : Les valeurs typiques de deze et de die (tableau basé sur les analyses présentées dans Kirsner 1979, 1993)

La différence en termes de force instructionnelle du SNdém dans les deux langues permet de mettre en perspective la fréquence relative nettement plus élevée des SNdém anaphoriques et mémoriels en français par rapport au néerlandais, d'expliquer des différences distributionnelles telles que ces dernières années vs. *deze laatste jaren et cette année 2011 vs. *dit jaar 2011 et de comprendre la fréquence malgré tout assez élevée de séquences à première vue redondantes telles que ${ }^{*} c e$ présent ouvrage sur Google. Ainsi, le fait d'avoir trouvé plusieurs exemples de SNdém anaphoriques français traduits par un SNdéf anaphorique en néerlandais (20), mais aucun cas de SNdém anaphorique néerlandais traduit par un SNdéf anaphorique français dans notre corpus s'explique par le fait que le contraste entre la force instructionnelle du SNdém anaphorique français (ce $\mathrm{N}$; cet édifice) et celle du SNdéf anaphorique néerlandais (de $N$; het gebouw) est moins grand que le contraste entre la force instructionnelle du SNdém anaphorique néerlandais introduit par une forme proximale (deze $N$; dit gebouw) et celle du SNdéf anaphorique français (le $N$; l'édifice), étant donné que le SNdéf anaphorique français (le $N$; l'édifice) a une force instructionnelle inférieure au SNdéf anaphorique néerlandais (de $N$; het gebouw) pour récupérer son référent dans le co-texte antérieur et que le SNdém anaphorique français (ce $N$; cet édifice) a une force référentielle inférieure ou égale au SNdém anaphorique néerlandais introduit par une forme proximale (deze $N$; dit gebouw).

(20) Inscrit entre ville et littoral, bordé par le nouveau projet urbain Neptune, le LAAC (Lieu d'art et d'action contemporaine) a rouvert ses portes le 25 juin 2005, rénové par les architectes Grafteaux \& Klein, qui ont su très subtilement transformer les défauts de cet édifice en qualité : éclairage, acoustique et mobilier. 
Op 25 juni 2005 opende het LAAC (Lieu d'art et d'action contemporaine) opnieuw zijn deuren. Het werd gerenoveerd door de architecten Grafteaux \& Klein, die de oorspronkelijke tekortkomingen van het gebouw op een subtiele manier tot troeven wisten om te vormen (verlichting, akoestiek en meubilair).

(DPC - Textes informatifs)

De plus, pour ce qui est de l'emploi mémoriel du SNdém, l'analyse qualitative de nos exemples, comme l'exemple (21), montre que le SNdém mémoriel français (ce $N$; ces fleurs ...), sollicitant des connaissances partagées, se rapproche davantage des SNdéf mémoriels français (le $N$; les fleurs ...) et néerlandais (de $N$; de droogbloemen ...), qui présupposent tout simplement les connaissances partagées, à cause de sa force instructionnelle affaiblie, contrairement au SNdém mémoriel néerlandais (deze / die $N$; deze / die droogbloemen ...), qui sollicite non seulement les connaissances partagées, mais qui supposerait également la présence presque immédiate du référent dans le cotexte antérieur ou dans le contexte situationnel.

(21) Sa silhouette fine, haute et droite, son visage mince aux traits réguliers, un peu creusés par l'âge, presque durs, faisaient penser à ces fleurs desséchées dont la beauté ancienne demeure visible dans la raideur fanée de la mort.

Haar tengere, lange en kaarsrechte gestalte, haar smalle, regelmatige, door de naderende ouderdom enigszins ingevallen, bijna harde gezicht deed denken aan de droogbloemen waarvan de vroegere schoonheid door de verwelkte starheid van de dood heen zichtbaar blijft.

(CdN - Textes fictionnels)

Si le SNdém mémoriel néerlandais introduit par une forme distale renvoie parfois de manière trop directe au co- et/ou contexte, ce renvoi est encore beaucoup plus intense pour le SNdém mémoriel néerlandais introduit par une forme proximale. En effet, la forme démonstrative proximale donne une instruction très forte pour trouver son référent dans le co- et/ou contexte, ce qui fait que son emploi n'est souvent pas approprié pour l'emploi mémoriel. Dans le même sens, De Mulder - Carlier (2006 : 109) notent que le démonstratif de proximité marque en général « que les informations disponibles dans le contexte immédiat de son occurrence - énonciatif ou textuel suffisent pour identifier le référent, alors que le démonstratif de distance suggère que d'autres informations que celles disponibles dans le contexte immédiat de son occurrence contribuent au repérage du référent ». Le démonstratif de distance fait donc à la fois appel à des informations présentes dans le co- et/ou contexte, mais également à des connaissances partagées dans la mémoire. Voilà pourquoi nous avons trouvé tant de SNdém mémoriels néerlandais introduits par les formes distales die et dat dans notre corpus et que l'emploi des formes proximales deze et dit est moins approprié pour l'emploi mémoriel. En français, l'opposition entre la proximité et la distance au niveau du déterminant démonstratif n'est pas marquée, ce qui constitue bien sûr une autre composante - structurelle - importante de l'affaiblissement de sa force instructionnelle. Cela montre à quel point il est indispensable d'inclure les différences structurelles entre les deux systèmes démonstratifs en français et en néerlandais dans la comparaison de leurs emplois référentiels.

Enfin, nos données montrent que la force instructionnelle véhiculée par le déterminant démonstratif dans certains SNdém temporels et déictiques textuels purs diffère 
sensiblement en français et en néerlandais (22), celle des déterminants démonstratifs néerlandais étant en effet trop élevée et provoquant un effet pléonastique. Le déterminant démonstratif est, par conséquent, remplacé par l'article défini en néerlandais.

(22) De algemene Vlaamse cultuur was in de laatste decennia weinig stedelijk. Ces dernières décennies, la culture générale flamande n'était guère urbaine. (DPC - Textes non-fictionnels)

En conclusion, les diverses données nous mettent sur la voie de la désémantisation du SNdém français et, par conséquent, sur le rapprochement du déterminant démonstratif et de l'article défini en français. Bien que le déterminant démonstratif français ne marque plus l'opposition proximité - distance et que sa force instructionnelle soit affaiblie dans plusieurs emplois référentiels, nous soutenons cependant avec De Mulder - Carlier (2006) que le déterminant démonstratif français n'a pas encore acquis le statut de l'article défini français, contrairement à l'hypothèse de Harris (1977). En effet, même si le SNdém mémoriel français se rapproche, par exemple, parfois de manière très étroite du SNdéf mémoriel français, les exemples montrent que l'opposition entre sollicitation et présupposition de connaissances partagées continue à être une opposition valable permettant de distinguer les deux cas.

\section{Conclusion}

Dans cet article, nous avons proposé un nouvel aperçu synthétique et unifié des emplois référentiels du SNdém qui revêt la forme d'un triangle. Cet aperçu, que nous avons réalisé au moyen d'une étude critique de la littérature en interaction avec une analyse qualitative d'exemples authentiques, ne tient pas seulement compte des emplois majeurs du SNdém, comme l'anaphore fidèle et l'emploi situationnel du SNdém, mais également de ses emplois moins courants, tels que l'emploi générique de sous-espèce et certains emplois textuels métalinguistiques ou métadiscursifs. Notre aperçu a également essayé de fournir une réponse au problème de la définition et de la délimitation de la deixis discursive et de l'emploi mémoriel, ainsi qu'à la dichotomie définitude sémantique - pragmatique. Par ailleurs, l'intégration des différents emplois attestés dans la littérature et la clarification de certains problèmes terminologiques n’étaient pas la seule motivation pour créer un nouvel aperçu des emplois référentiels du SNdém. Il devait aussi servir de point de départ à l'étude contrastive basée sur corpus des emplois référentiels du SNdém en français et en néerlandais contemporains. Grâce à la représentation multidimensionnelle des différents emplois du SNdém, nous avons pu montrer que les SNdém français sont plus fréquents que les SNdém néerlandais dans la partie inférieure de l'aperçu, partie qui se rapproche du pôle de la récupération nonphorique ou générique. Nous avons ensuite mis cette constatation en rapport avec l'hypothèse de l'affaiblissement de la force instructionnelle du SNdém français, en fournissant de nouvelles preuves concrètes issues de la linguistique contrastive synchronique et en précisant en quoi consiste exactement la désémantisation du SNdém français, à savoir sa force instructionnelle affaiblie. 


\section{Références bibliographiques}

Apothéloz, D. (1995). Rôle et fonctionnement de l'anaphore dans la dynamique textuelle. Genève : Droz. Ariel, M. (1988). Referring and Accessibility. Journal of Linguistics, 24, 65-87.

van Baardewijk-Rességuier, J. - van Willigen-Sinemus, M. (1989). Matériaux pour la traduction du néerlandais en français. Muiderberg : Coutinho. [2 tomes]

Biber, D. - Johansson, S. - Leech, G. - Conrad, S. - Finegan, E. (1999). Longman Grammar of Spoken and Written English. Essex : Pearson ESL.

Breban, T. (2010). The PDE and Historical Functions of That in the NP. Communication au 43rd Annual Meeting Societas Linguistica Europaea (2-5 septembre 2010, Vilnius, Lithuanie).

Christophersen, P. (1939). The Articles : A Study of Their Theory and Use in English. København: Munksgaard.

Corblin, F. (1983). Défini et démonstratif dans la reprise immédiate. Le français moderne, 51, 118-134.

Cornish, F. (1999). Anaphora, Discourse and Understanding. Evidence from English and French. Oxford : Clarendon Press.

Cornish, F. (2000). L'accessibilité cognitive des référents, le centrage d'attention et la structuration du discours : une vue d'ensemble. Verbum, 22 (1), 7-31.

Cornish, F. (2001). Modal That as Determiner and Pronoun: The Primacy of the Cognitive-Interactive Dimension. English Language and Linguistics, 5 (2), 297-315.

Cornish, F. (2007). English Demonstratives : Discourse Deixis and Anaphora. In : Nilsen, R.A. - AppiahAmfo, N.A. - Borthen, K. (eds). Interpreting Utterances : Pragmatics and Its Interfaces. Essays in Honour of Thorstein Fretheim. Oslo : Novus Press. 147-166.

Cornish, F. (2008). How Indexicals Function in Texts : Discourse, Text, and one Neo-Gricean Account of Indexical Reference. Journal of Pragmatics, 40 (6), 997-1018.

Cornish, F. (2010). Strict Anadeixis and Discourse Deixis via Demonstratives: Textual Signals of Discourse Structure. Communication au $43^{\text {rd }}$ Annual Meeting Societas Linguistica Europaea (2-5 septembre 2010, Vilnius, Lithuanie).

Davis, C. - Potts, C. (2009). Affective Demonstratives and the Division of Pragmatic Labor. In : Aloni, M. - Bastiaanse, H. - de Jager, T. - van Ormondt, P. - Schulz, K. (eds). Preproceedings of the 17th Amsterdam Colloquium. 32-41.

Demol, A. (2010). Les pronoms anaphoriques celui-ci et il. Bruxelles : De Boeck - Duculot.

De Mulder, W. (1997). Les démonstratifs : des indices de changement de contexte. In : Flaux, N. - Van de Velde, D. - De Mulder, W. Entre général et particulier : les déterminants. Arras : Artois Presses Université. 137-200.

De Mulder, W. - Carlier, A. (2006). Du démonstratif à l'article défini : le cas de ce en français moderne. Langue française, 152, 96-113.

Devos, F. - De Muynck, R. - Van Herreweghe, M. (1991). Nederlands, Frans en Engels in Contrast (tome 1). Leuven : Peeters.

Diessel, H. (1999). Demonstratives : Form, Function and Grammaticalization. Amsterdam / Philadelphia : John Benjamins.

Ehlich, K. (1982). Anaphora and Deixis. Same, Similar, or Different ?. In : Jarvella, R.J. - Klein, W. (eds). Speech, Place and Action : Studies in Deixis and Related Topics. New York : Wiley. 315-338.

Epstein, R. (1994). The Development of the Definite Article in French. In: Pagliuca, W. (éd.). Perspectives on Grammaticalization. Amsterdam / Philadelphia : John Benjamins. 63-80.

Fillmore, C.J. (1997). Lectures on Deixis. Stanford : CSLI.

Gary-Prieur, M.-N. - Noailly, M. (1996). Démonstratifs insolites. Poétique, 105, 111-121.

Goethals, P. (2007). Corpus-Driven Hypothesis Generation in Translation Studies, Contrastive Linguistics and Text Linguistics : A Case Study of Demonstratives in Spanish and Dutch Parallel Texts. Belgian Journal of Linguistics, 21, 87-103.

Grevisse, G. - Goosse, A. 201115. Le Bon Usage. Bruxelles : De Boeck - Duculot.

Grosz, B.J. - Joshi, A.K. - Weinstein, S. (1995). Towards a Computational Theory of Discourse Interpretation. Computational Linguistics, 21 (2), 203-225. 
Guillot, C. (2003). Le rôle des démonstratifs dans la cohésion textuelle au XVe siècle. Éléments de grammaire textuelle. Paris : ENS. [Thèse de doctorat]

Gundel, J.K. - Hedberg, N. - Zacharski, R. (1993). Cognitive Status and the Form of Referring Expressions in Discourse. Language, 62 (2), 274-307.

Greenberg, J.H. 1978. How Does a Language Acquire Gender Markers ?. In : Greenberg, J.H. Ferguson, C.A. - Moravcsik, E.A. (eds). Universals of Human Language (tome 3). Stanford : Stanford University Press. 47-82.

Haeseryn, W. - Romijn, K. - Geerts, G. - De Rooij, J. - Van den Toorn, M.C. (1997). Algemene Nederlandse Spraakkunst. Groningen : Wolters - Noordhoff.

Halliday, M.A.K. - Hasan, R. (1976). Cohesion in English. London : Longman.

Harris, M. (1977). Demonstratives, Articles and Third Person Pronouns in French : Changes in Progress. Zeitschrift für Romanische Philologie, 93, 249-261.

Hawkins, J. (1978). Definiteness and Indefiniteness: A Study in Reference and Grammaticality Prediction. London : Croom Helm.

Himmelmann, N.P. (1996). Demonstratives in Narrative Discourse : A Taxonomy of Universal Uses. In : Fox, B. (ed.). Studies in Anaphora. Amsterdam / Philadelphia : John Benjamins. 205-254.

Jonasson, K. (1998). Le déterminant démonstratif : un marqueur de quoi ?. Travaux de linguistique, 36, 59-70.

Kirsner, R.S. (1979). Deixis in Discourse : An Exploratory Quantitative Study of the Modern Dutch Demonstrative Adjectives. In: Givón, T. (ed.). Syntax and Semantics (tome 12). New York: Academic Press. 355-375.

Kirsner, R.S. (1993). From Meaning to Message in Two Theories : Cognitive and Saussurean Views of the Modern Dutch Demonstratives. In : Geiger, R.A. - Rudzka-Ostyn, B. (eds). Conceptualizations and Mental Processing in Language. Berlin : Mouton de Gruyter. 81-114.

Kleiber, G. (1984). Sur la sémantique des descriptions démonstratives. Lingvisticae Investigations, 8 (1), 63-85.

Kleiber, G. (1986). Déictiques, embrayeurs, token-réflexives, symboles indexicaux, etc. : comment les définir ?. L'information grammaticale, 30, 3-22.

Kleiber, G. (1987). L’opposition cist / cil en ancien français, ou comment analyser les démonstratifs ?. Revue de linguistique romane, 51, 5-35.

Kleiber, G. (1994). Qu'est-ce qui est (in)défini ?. Faits de langues, 4, 81-87.

Kleiber, G. (2005). Des démonstratifs bien énigmatiques : les démonstratifs cataphoriques génériques. In : Dobrovie-Sorin, C. (éd.). Noms nus et généricité. Saint-Denis: Presses Universitaires de Vincennes. 65-95.

Kleiber, G. (2008). Démonstratifs marginaux : lorsque le démonstratif se met en titre. Communication à la Journée d'Étude La détermination côté définitude (le 28 mai 2008, Lille, France).

Kleiber, G. - Lazzaro, H. (1987). Qu'est-ce qu'un syntagme nominal générique ? Ou Les carottes qui poussent ici sont plus grosses que les autres. In : Kleiber, G. (éd.). Rencontre(s) avec la généricite. Paris : Klincksieck. 73-111.

Kleiber, G. - Sock, R. (2006). Ces + N + relative : sémantique et prosodie. Lingvisticae investigationes, 29 (2), 251-273.

Korzen, I. - Lundquist, L. (éds). (2003). Sprogtypologi og Oversaettelse. Endocentriske og Exocentriske Sprog. København : Samfundslitteratur.

Lakoff, R. (1974). Remarks on This and That. In : La Galy, M.W. - Fox, R.A. - Bruck, A. (eds). Papers from the $10^{\text {th }}$ Regional Meeting of the Chicago Linguistic Society. Chicago: Chicago Linguistic Society. 345-356.

Leeman, D. (2004). Les déterminants du nom en français. Paris : Presses Universitaires de Paris.

Levinson, S.C. (1983). Pragmatics. Cambridge : Cambridge University Press.

Levinson, S.C. (2004). Deixis. In : Horn, L.R. - Ward, G. (eds). The Handbook of Pragmatics. Oxford : Blackwell. 97-121.

Löbner, S. (1985). Definites. Journal of Semantics, 4, 279-326.

Lundquist, L. (2005). Noms, verbes et anaphores (in)fidèles. Pourquoi les Danois sont plus fidèles que les Français. Langue française, 145, 73-91. 
Lyons, J. (1977). Semantics. Cambridge : Cambridge University Press.

Lyons, C. (1999). Definiteness. Cambridge : Cambridge University Press.

Maes, A. (1996). Nominal Anaphors, Markedness and the Coherence of Discourse. Leuven : Peeters.

Marchello-Nizia, C. (2006). Grammaticalisation et changement linguistique. Bruxelles : De Boeck Duculot.

Paulussen, H. (1999). A Corpus-Based Contrastive Analysis of English on / up, Dutch op and French sur within a Cognitive Framework. Gent : Universiteit Gent. [Thèse de doctorat]

Paulussen, H. - Macken, L. - Trushkina, J. - Desmet, P. - Vandeweghe, W. (2006). Dutch Parallel Corpus : A Multifunctional and Multilingual Corpus. Cahiers de l'Institut de Linguistique de Louvain, 32 (1) (2) (3) (4), 269-285.

Philippe, G. (1998). Les démonstratifs et le statut énonciatif des textes de fiction : l'exemple des ouvertures de roman. Langue française, 120, 51-65.

Reichler-Béguelin, M.-J. (1995). Alternatives et décisions lexicales dans l'emploi des expressions démonstratives. Pratiques, 85, 53-87.

Riegel, M. - Pellat, J.-C. - Rioul, R. $\left(2009^{4}\right)$. Grammaire méthodique du français. Paris: Presses Universitaires de France.

Searle, J. (1969). Speech Acts: An Essay in the Philosophy of Language. Cambridge : Cambridge University Press.

Skytte, G. - Korzen, I. (2000). Italiensk-Dansk Sprogbrug i Komparativt Perspektiv. København : Samfundslitteratur.

Van Belle, W. - Lamiroy, B. - Van Langendonck, W. - Lahousse, K. - Lauwers, P. - Van Goethem, K. (2005). Nederlandse Grammatica voor Franstaligen. Leuven : Katholieke Universiteit Leuven.

Van Langendonck, W. (1976). Definiteness, Genericness and Markedness. Leuven : Katholieke Universiteit Leuven. [Preprint 39]

Whittaker, S. (2004). Étude contrastive des syntagmes nominaux démonstratifs dans des textes traduits du français en norvégien et des textes sources norvégiens: stratégie de traduction ou translationese ?. Forum, International Journal of Interpretation and Translation, 2 (2), 221-239.

Wilmet, M. (1997). Grammaire critique du français. Bruxelles : De Boeck - Duculot.

${ }^{1}$ E.a. Grevisse - Goosse $\left(2011^{15}\right)$ pour le français, Biber et al. (1999) pour l'anglais, Haeseryn et al. (1997) pour le néerlandais.

${ }^{2}$ E.a. Chrisophersen (1939), Kleiber (1994), Lyons (1999), Riegel et al. $\left(2009^{4}\right)$.

${ }^{3}$ E.a. Searle (1969), Hawkins (1978).

${ }^{4}$ E.a. Van Langendonck (1976) pour une analyse en termes de marquage.

${ }^{5}$ E.a. Corblin (1983, Kleiber (1984), Maes (1996), Wilmet (1997), Leeman (2004).

${ }^{6}$ E.a. les synthèses de De Mulder (1997), de Cornish (2000) et de Demol (2010) sur la Théorie de l'Accessibilité (e.a. Ariel 1988), la Hiérarchie du Donné (e.a. Gundel et al. 1993) et la Théorie du Centrage (e.a. Grosz et al. 1995).

${ }^{7}$ E.a. Harris (1997), Epstein (1994), Kleiber (1987), Guillot (2003), De Mulder - Carlier (2006), Marchello-Nizia (2006) pour le français.

${ }^{8}$ E.a. Reichler-Béguelin (1995), Himmelmann (1996), Diessel (1999), Levinson (2004), Cornish (2008).

${ }^{9}$ E.a. Lakoff (1974), Apothéloz (1995), Kleiber (1986), Breban (2010).

${ }^{10}$ E.a. Gary-Prieur - Noailly (1996), Kleiber - Lazzaro (1987), Philippe (1998), Kleiber - Sock (2006).

${ }^{11}$ E.a. Jonasson (1998) : français - suédois, Whittaker (2004) : français - norvégien, Goethals (2007) : espagnol néerlandais.

${ }^{12}$ E.a. van Baardewijk-Rességuier - van Willigen-Sinemus (1989), Devos et al. (1991), Van Belle et al. (2005) pour le français et le néerlandais.

13 " Languages in Stage II generally include instances of non-referential use so that they correspond grosso modo to the combined uses of a definite and indefinite article. » (Greenberg 1978 : 62-63) 